\title{
HERRAMIENTAS DIGITALES
}

PARA FACILITAR EL ACCESO A ESPACIOS DE PARTICIPACIÓN CIUDADANA EN LA FORMULACIÓN DE POLITICAS PÚBLICAS

DIGITAL TOOLS TO FACILITATE ACCESS TO DIGITAL CITIZEN PARTICIPATION PLATFORMS DURING THE FORMULATION OF PUBLIC POLICIES

\section{Bryan Franco Sarasty}

Programa de diseño de medios interactivos,

Departamento de diseño, Universidad Icesi. bryan.franco.sarasty@ gmail.com.

\section{Javier Aguirre Ramos}

Programa de diseño de medios interactivos, Departamento de diseño, Universidad Icesi.

09 edu.co. 
Palabras clave

Gobierno digital,

participación digital,

democracia digital,

participación ciudadana

política pública.

\section{Resumen}

Utilizando la metodología de doble diamante en diseño, generamos una propuesta de herramienta digital para facilitar el acceso a espacios de participación ciudadana durante la formulación de políticas públicas. Esto, soportado sobre una base teórica que da cuenta del estado de la participación ciudadana en el contexto colombiano, las dificultades que enfrenta la digitalización en el país y las características de los espacios de participación; así como una base empírica sustentada en los aportes de expertos en la materia y el análisis de las plataformas de participación ciudadana digital existentes. Finalmente, con base en lo expuesto con anterioridad, evidenciamos las determinantes y requerimientos que determinan el funcionamiento de nuestra propuesta.

\section{Abstract}

Using the double diamond methodology in design, we generated a digital tool to facilitate access to citizen

participation platforms during public policy formulation. This, supported on a theoretical basis that gives an account of the state of citizen participation in the Colombian context, the difficulties faced by digitization in the country and the

characteristics of its digital citizen platforms; as well as an empirical basis built on input from subject matter experts and an analysis of existing digital citizen participation platforms. Finally, we show the determinants and requirements that establish the inner workings of our proposal. 
avance en el proyecto y se produjeron tres propuestas a ser discutidas posteriormente por el Concejo de Cali. Sin embargo, a pesar de este progreso, la ciudadanía aún desconoce en buena medida las implicaciones particulares de Cali Distrito Especial, más allá de los postulados generales sobre nuevas estructuras administrativas. Del mismo modo, es de resaltar que en una ciudad de dos millones de habitantes, tan sólo 665 personas participaron de los espacios abiertos por la alcaldía, la mayoría citadas por ser pertenecientes a organizaciones y comunidades ya estructuradas, usualmente en contacto con temas con relación a lo público.

Tras esta realidad se hallan serios vacíos en la participación ciudadana y el acceso a sus procesos durante el desarrollo e implementación de políticas públicas como Cali Distrito Especial. Los espacios de participación están cubiertos por una nube de desconocimiento, lo cual lleva a una participación de los ciudadanos reducida o limitada por la poca información que se tiene acerca de su funcionamiento, así como los asuntos que en ellos se discuten (Velásquez et. al, 2019b)

Aunque la Constitución Política de Colombia consagra siete mecanismos de participación ciudadana institucionalizados, tan sólo el cabildo abierto cuenta con facultades de deliberación y discusión progresiva. Factor que lo hace ideal para la construcción de políticas públicas y la discusión de decisiones administrativas. Sin embargo, también es el mecanismo de mayor desconocimiento por la ciudadanía, con un $72 \%$ de ciudadanos con más de 18 años que no sabe de su existencia y funcionamiento (DANE, 2019). Del mismo modo, el cabildo abierto es un mecanismo de participación que sólo puede convocarse por iniciativa ciudadana, es decir, con la solicitud oficial de un grupo de ciudadanos. Esto es problemático por dos razones. En primer lugar, la ciudadanía no puede dar inicio a procesos que desconoce en su mayoría, lo que constriñe en buena medida la posibilidad de hacer escuchar su voz. Por otro lado, la solicitud debe pasar por un proceso de aprobación que puede tomar un tiempo considerable, hecho que rompe la continuidad de la iniciativa.

En el caso de la participación convocada por tomadores de decisiones, los problemas son los mismos. Aunque no existen mecanismos de participación ciudadana institucionalizados donde la iniciativa sea de actores estatales, es natural que las administraciones se destacaron líderes de grupos y comunidades diversas (Alcaldía de Cali, 2019). Como producto de la información recogida hubo un gran 
locales dispongan de espacios para realizar procesos participativos como talleres y grupos focales, tal y como evidencia la experiencia de Cali Distrito Especial. No obstante, en ellos se incurre en la misma problemática, esto es: Los ciudadanos no tienen el tiempo para participar y cuando lo hacen no saben cómo funcionan tales espacios, qué políticas se discuten en ellos, cuál es su incidencia y si son vinculantes o no (Velásquez et. al, 2019b).

En síntesis, se podría considerar que más allá de un problema de información, esta es una cuestión de acceso limitado a los procesos de participación ciudadana. En otras palabras, es el producto de una limitación en el involucramiento dentro de los espacios de participación dada por su funcionamiento tradicional, el cual suele demandar sincronicidad en los encuentros, lo que imposibilita la intervención de ciudadanos con poco o nada de tiempo disponible. Del mismo modo, este último factor es un límite en la comprensión de los procesos y las políticas en discusión que constriñe el acceso pleno al espacio de participación ciudadana, pues las intervenciones de ciudadanos desinformados usualmente escapan al tema en cuestión y tienen poca incidencia en los diagnósticos establecidos (Velásquez et. al, 2019a).

Ante problemáticas similares, se ha iniciado una tendencia global de implementación de herramientas digitales a modo de romper con las limitaciones de espacio y tiempo fijos con el fin de crear espacios de participación ciudadana ya no necesariamente presenciales, pero efectivos en la virtualidad (Sanabria, Pliscoff y Gomes, 2014). La inserción de los medios digitales en el día a día ha modificado la relación entre ciudadano y tomador de decisiones, así como ha facilitado la difusión de información concerniente a lo público (Dunleavy, Margetts, Bastow y Tinkler, 2006; Velásquez et al, 2019c). Tales prácticas tienen como facultad la posibilidad de generar procesos de participación con mayor alcance y carácter asincrónico, lo cual lleva a preguntarse: ¿Cómo desarrollar una herramienta digital que facilite el acceso a espacios de participación ciudadana en el desarrollo e implementación de políticas públicas?

\section{Marco Teórico}

Desarrollar una herramienta digital que aborde el acceso a espacios de participación implica, de manera imperativa, conocer las categorías analíticas que se relacionan con el tema. Aunque la literatura en esta área es amplia, es necesario delimitar los conceptos que la componen a modo de facilitar su comprensión. Es por esto que a continuación se recorre la literatura relacionada con espacios de participación de carácter digital y se analiza en función a la presente investigación.

\section{Del gobierno a la democracia digital}

El escenario político no ha sido ajeno a la revolucionaria transformación

tecnológica que se ha dado en los últimos 20 años. Con el surgimiento y proliferación de nuevas tecnologías de la información y la comunicación (TIC), particularmente el internet, gobiernos de todo el mundo se están embarcando en la tendencia de incorporar estos nuevos paradigmas a sus sistemas políticos. Actividad generalmente adscrita a conceptos como democracia (Holzer, Melitskin, Rho y Schwester, 2004) o gobierno digital.

Si bien, ambas ideas implican el empleo de las tecnologías de la información y la comunicación (TIC) en los procesos y actividades de carácter público, en la literatura sobre el tema existe un debate sobre los atributos que marcan la diferencia entre ellas. Para algunos democracia y gobierno digital son ideas con grandes distinciones, únicamente conectadas por la implementación de las TIC, mientras que otros defienden su alusión a los mismos fenómenos (Kardan y Sadeghiani, 2011). A pesar del amplio avance tecnológico y la aplicación empírica de la tecnología en el sector público, aún no existe un consenso para la discusión. Sin embargo, es común encontrar al gobierno digital conceptualizado como condición necesaria mas no suficiente para el alcance de la democracia digital. En otras palabras, implementar las TIC en el ejercicio del gobierno no es garantía del traslado de los valores y prácticas democráticas al espacio digital, pero es absolutamente necesario si se quiere lograr tal propósito.

Una buena parte del gobierno digital se concentra en procesos electorales o partidistas en el que los candidatos tienen como propósito comunicarse con sus electores de manera directa, lo cual potencia la confusión al momento de hacer la distinción entre ambos conceptos (Netchaeva, 2002). Shackleton, Fisher y Dawn (2004) nos presentan al e-gobierno como un proceso de tres etapas a desarrollar para alcanzar su madurez definitiva. En primer lugar, se encuentra el gobierno digital como la simple presencia de las TIC en la gestión pública; posteriormente, se alcanza la etapa de gobernanza digital, materializada 
en la provisión de servicios públicos y el establecimiento de formas digitales de democracia representativa; finalmente, se llega a un punto de e-democracia en el que la ciudadanía tiene la capacidad de participar directamente en espacios de decisión.

En este orden de ideas, el gobierno digital puede caracterizarse como una aplicación paulatina de las tecnologías de la información y la comunicación a las diferentes prácticas y actividades que constituyen la labor gubernamental con el objetivo de proveer servicios más eficaces y eficientes (Kardan y Sadeghiani, 2011) o una transferencia de las actividades gubernamentales hacia un modelo digital (Netchaeva 2002); mientras que la democracia digital puede ser asociada a la vigorización de los procesos democráticos como producto de la misma. Así, entre las aplicaciones del e-gobierno que producen e-democracia pueden ser considerados el uso de plataformas digitales o la digitalización de instancias ya existentes en pro del mejoramiento de la rendición de cuentas de los funcionarios públicos, el incremento de la transparencia en el ejercicio político, la facilitación de los procesos electorales y, como compete a la presente investigación, la facilitación del acceso a espacios de participación ciudadana.

El gran potencial del e-gobierno se halla en atributos como la interactividad, la conectividad, la libertad de expresión y asociación, la construcción y diseminación de información y la puesta a prueba de las perspectivas oficiales. Ante la concepción del Estado como un ente distante y críptico, el gobierno digital tiene el poder de sobrepasar los límites que generan los criterios rígidos y la inflexibilidad dada por un tiempo y espacio fijos. Hecho que permite la interacción directa entre ciudadanos y tomadores de decisiones (Hague y Loader, 2005). En otras palabras, el gobierno digital tiene la capacidad de democratizar procesos, ya que traslada al ciudadano del rol de receptor pasivo al de individuo activo en el escenario político. Se trata de expandir el ejercicio democrático más allá del voto, promoviendo la participación ciudadana en otros espacios con mayor campo de acción.

\section{Gobierno y datos abiertos}

Hacer del gobierno una actividad más democrática implica implementar modelos que permitan su expansión hacia sectores que, de un modo u otro, previamente no eran capaz de alcanzar. Entre las prácticas que acercan a las diferentes administraciones al cumplimiento de tal propósito se hallan el gobierno y datos abiertos, conceptos ampliamente adoptados en la literatura sobre e-gobierno. Su estrecho vínculo hace que hablar de uno de los conceptos lleve, usualmente, hacia la mención del otro. Esto no es sorpresa, si se considera que la construcción de ambas ideas se ha dado en términos similares. Sin embargo, su ambigüedad produce problemas al momento de aplicarlos a la realidad pues la falta de claridad conlleva a la difícil evaluación y delimitación de lo que se pretende lograr con ellos (Yu y Robinson, 2012).

Es necesario establecer sus diferencias con el propósito de entender qué implicaciones tienen sobre el gobierno y la democracia digital. La Carta Internacional de Datos Abiertos (2015) establece que "los datos abiertos son datos hechos disponibles con las características técnicas y legales necesarias para ser usados, reusados y redistribuidos de manera libre por cualquiera en cualquier momento o espacio". Definición que, en términos generales, se asocia con la de gobierno abierto en cuanto a que este es usualmente concebido como una aplicación de datos abiertos en la labor gubernamental (Yu y Robinson, 2012). Sin embargo, esta conceptualización es tan sólo parcialmente correcta, pues más allá de datos accesibles el gobierno abierto implica transparencia, responsabilidad y participación ciudadana como formas de control y revisión del sector público (Ingrams, Piotrowski y Berliner, 2020).

Así, el gobierno abierto se perfila como una práctica que instrumentaliza los datos abiertos a modo de facilitar la transparencia y accesibilidad a espacios de decisión para los ciudadanos. Su principio fundamental se halla entonces en el uso de la información para la acción democrática. No se trata únicamente de distribuir datos para su lectura y asimilación, sino que demanda un uso de esta información para participar e incidir en el escenario público.

\section{Los retos y posibilidades del gobierno digital}

Numerosas iniciativas de gobierno digital suelen quedarse atascadas sin alcanzar la etapa de e-democracia a pesar de los esfuerzos realizados para lograr su prevalencia. Bolgherini (2007) estudia las razones para el fracaso o el éxito del e-gobierno y reitera su insuficiencia para alcanzar una democracia real. Admite su gran potencial, pero demuestra que sólo una pequeña minoría de iniciativas de gobierno digital terminan siendo adoptadas por la ciudadanía y cumpliendo con su propósito final. 
El problema del gobierno digital se halla en su origen mismo. La iniciativa nace desde el Estado, es este quien decide implementar las TICs en su labor y, por lo tanto, es quien determina el punto de partida y los límites del gobierno digital. Hay tanto espacio para involucrarse como los tomadores de decisiones abran. Es en ese punto donde las promesas del e-gobierno pierden credibilidad. Aunque se resalta la ruptura de jerarquías como una de sus bondades, en la práctica se mantienen (Bolgherini, 2007).

Netchaeva (2002) habla de una falta de tradición de intercomunicación y discusión pública que bloquea el uso fructífero de los sitios gubernamentales. En otras palabras, existen pocos referentes que guíen la construcción de un gobierno digital. Aún cuando la digitalización del ejercicio gubernamental parece facilitar la labor de actores políticos como ONGs y gobiernos, los ciudadanos se ven beneficiados en menor medida por estas transformaciones. Para los primeros se hace más sencillo alcanzar al ciudadano, proveer información e incluso publicitarse; mientras que los últimos suelen mantenerse en una posición pasiva aunque el propósito sea distinto (Netchaeva, 2002).

Ahora bien, Thomas y Streib (2003) contemplan la posibilidad de contactos con el gobierno iniciados por ciudadanos. Afirman que existe la posibilidad de que la ciudadanía tome la iniciativa de exigir que el gobierno abra espacios de participación ciudadana destinados a sus temas de interés. No obstante, toman como supuesto que estos acercamientos se darán por medio de sitios web provistos por el gobierno. Su idea de ciudadanos que proactivamente buscan la interacción con los tomadores de decisiones es acertada. Esta última, materializada en la actualidad puede servir para imaginar espacios que, aprovechando el carácter libre y flexible de las nuevas tecnologías, sean iniciadas por actores distintos al gobierno. Es en este punto en el que la presente investigación busca intervenir.

\section{Participación ciudadana, el vínculo faltante}

¿De qué depende que el gobierno digital produzca democracia digital? La respuesta a esta pregunta se encuentra inmersa en la previamente mencionada idea de gobierno digital como proceso de tres instancias, particularmente en su segunda fase, esto es: La gobernanza digital. En su análisis, Shackleton et. al (2004) la caracterizan como un momento intermedio de la transición desde gobierno digital a democracia digital, en el que el uso de las TIC empieza a naturalizarse y genera ligeros acercamientos a procesos que si bien son más democráticos, aún no cuentan con el involucramiento directo de la ciudadanía.

Es en este último punto en el que se halla el elemento que propicia o restringe el surgimiento de la democracia digital. Bajo esta definición, la democratización de la gestión pública sólo lleva hacia una e-democracia si cuenta con la introducción de la ciudadanía de manera directa en estos procesos. Hecho que parece indicar que el vínculo que conecta el gobierno y democracia digital es la participación ciudadana digital. Netchaeva (2002) se refiere al e-gobierno como una práctica insuficiente para alcanzar la democracia real, pues este logro depende de que todos los ciudadanos tengan la posibilidad de participar directamente en la toma de decisiones, comunicarse con quienes deciden y tener una opinión libre de presiones. En otras palabras, es fundamental que más allá de lograr procesos más eficientes y transparentes, se logre acercar a la ciudadanía a espacios de participación.

\section{La participación ciudadana}

Antes de analizar a profundidad el papel de la participación ciudadana en la ecuación que lleva del e-gobierno a la e-democracia, es fundamental entenderla bien. La participación ciudadana es:

Un proceso social que resulta de la acción intencionada de individuos y grupos en busca de metas específicas, en función de intereses diversos y en el contexto de tramas concretas de relaciones sociales y de poder" así como un "medio para la formulación, discusión y adopción de los grandes consensos sobre el desarrollo y la convivencia en el territorio (Velásquez y González, 2003, p. 19).

La participación ciudadana, como parte esencial de las democracias, tiene múltiples beneficios. En primer lugar, permite generar dar visibilidad a las posiciones de los ciudadanos con el propósito de alcanzar un consenso, reduce las jerarquías y facilita el diálogo. Aunque tiene la capacidad de influenciar en buena medida la construcción de políticas públicas, depende también de la voluntad política y el involucramiento de los ciudadanos (Velásquez y González, 2003). Con 
el avance de la democratización, la participación ciudadana se ha popularizado como una forma de deliberación y decisión para distintas actividades en el sector público, entre las cuales destaca la planeación presupuestal participativa. Es un elemento que fortalece la democracia e incrementa la transparencia del sector público al involucrar directamente a los ciudadanos en estos procesos. Ahora bien, aunque es de gran importancia analizar la participación ciudadana como un concepto independiente, resulta profundamente valioso analizar los resultados de su interacción con las TIC. Al respecto, es importante tener en cuenta que estas son cada día más prevalentes en el día a día de la ciudadanía y, por tanto, podrían tener un poderoso efecto sobre la dinámica entre el e-gobierno y la e-democracia.

\section{Participación ciudadana digital}

La participación ciudadana digital o e-participación consiste, como su nombre insinúa, en crear nuevos espacios de participación ciudadana o trasladar los ya existentes a un entorno digital a través de las TIC (Peña-López, 2014). Su carácter virtual responde de manera eficaz a las limitaciones planteadas en la sección anterior y, en ese orden de ideas, cuenta con características que demandan un abordaje distinto. La gran facultad de la e-participación reside en que no se ve afectada en gran medida por restricciones de espacio y tiempo. El empleo de las TIC, particularmente el internet, facilita el acceso a espacios de manera casi inmediata en cualquier tiempo, sin restricciones que fuercen la sincronicidad de los actores involucrados (Peña-López, 2014). Así, dicho concepto tiene como propósitos principales la inclusión de los ciudadanos en la toma de decisiones de carácter público, la incidencia de estos en ellas, así como la concertación con los tomadores de decisiones y la vigilancia u oposición a las medidas que toman (Velásquez et al, 2019c).

Como medio, la participación ciudadana digital tiene las siguientes características (Velásquez et al, 2019c): Esta se encuentra ligada al concepto de globalización, en el sentido de que trasciende las fronteras y límites geográficos. Asimismo, se trata de un modelo segmentado que no extingue las formas de participación colectiva. Dicha propuesta, responde a la ausencia o baja visibilidad de espacios públicos existentes. Por último, por su carácter no presencial puede llegar a ser algo difusa por la velocidad y la flexibilidad en que se sustenta.
Al ser un concepto reciente, enfrenta grandes retos de delimitación, pues aún no son claros los atributos que deben tener las plataformas que la sustentan. Del mismo modo, no se sabe cómo varía su desempeño en diferentes contextos, quiénes son más propensos a utilizarlas, qué exactamente motiva su uso y de qué manera inciden en la toma de decisiones. No hay un consenso sobre la coherencia entre sus beneficios teóricos y su desempeño real. En términos generales, la participación ciudadana digital como producto de la integración entre el concepto tradicional de participación y la implementación de las TIC es una práctica con un poderoso alcance y capacidades. De tal modo, comprender que presenta grandes oportunidades para el avance hacia la democracia digital contextualiza la actual investigación y da luces sobre el abordaje que puede dársele al problema.

\section{Participación ciudadana en Colombia}

Estudiar la participación ciudadana digital no es tarea fácil. En la literatura se presentan numerosos contextos que en diferentes medidas parecen transformar sus efectos, características y maneras de proceder. De hecho, el caso colombiano no es la excepción: Si el propósito de la presente investigación es producir una herramienta para facilitar el acceso a espacios de participación ciudadana digital, es necesario conocer a cabalidad el contexto en el cual estará inmersa.

¿Por qué hablamos de espacios de participación ciudadana en Colombia? Aunque la constitución política estipula el cabildo abierto como medio para la deliberación y construcción de consensos entre ciudadanos y tomadores de decisiones, $72 \%$ de las personas adultas desconoce su existencia y jamás ha oído hablar de él. Ello implica que su empleo es notablemente reducido, lo que podría llevar a pensar que la participación ciudadana en Colombia es nula. Sin embargo, es usual que los gobiernos locales y la rama legislativa obedezcan a la ley y abran espacios para que la ciudadanía discuta temáticas de carácter público. Es por ello que hablar únicamente de mecanismos de participación sería insuficiente y desacertado, es decir, dejar de lado la iniciativa de los tomadores de decisiones sería cortar la forma de participación más prevalente en el país.

Con el cabildo abierto como única forma de deliberación estipulada y delimitada en la constitución, la participación ciudadana del país es notablemente irregular. Los distintos niveles de gobierno se 
valen de grupos focales, socializaciones y talleres con metodologías no estandarizadas que varían con cada iteración (Velásquez et al, 2019b), mientras que los mecanismos de participación consagrados en la constitución, como se mencionó anteriormente, tienen un carácter reactivo y movilizador que se traduce en veto a decisiones ya tomadas; tienen como requisito un umbral de firmas y usualmente deben ser convocados por ciudadanos.

La dificultad de la participación ciudadana en Colombia se halla en la reducida visibilidad que tienen los espacios deliberativos. La ciudadanía desconoce el cabildo abierto y cuando sabe de su existencia decide no convocarlo porque implica un gran esfuerzo realizarlo y cumplir con los requisitos. Por otro lado, si la iniciativa es impulsada por los tomadores de decisiones, usualmente los espacios se convierten en métodos de reafirmación o presión para aprobar determinada agenda política. En efecto, entre las razones por las cuales los ciudadanos no tienen acercamiento a estos, se encuentran las malas experiencias relacionadas con el sector público, la burocratización de los espacios, la baja identificación con ellos, la falta de tiempo y la cooptación política (Velásquez et al, 2019b).

La participación ciudadana digital también se encuentra rodeada por problemáticas. Aunque constituye un avance grande y logra superar los elementos que limitan a la participación ciudadana tradicional se ubica en un contexto poco amigable. Iniciativas como urna de cristal, a pesar de su buena intención, no alcanzan su propósito final por diferentes razones. En primer lugar, son plataformas iniciadas desde el gobierno, por lo que tienden a ser herramientas de confirmación de políticas ya existentes. En otras palabras, lo que allí se discuta se encuentra limitado a la decisión y provisión de los tomadores de decisiones. Por otro lado son plataformas de reducida visibilidad y utilización. En últimas, como consecuencia de la ya prevalente ausencia de espacios de participación tradicionales, las personas utilizan estas plataformas como medios de desahogo y compensación, en las que más allá de discutir los temas puestos sobre la mesa deciden hacer preguntas o afirmaciones sin relación con estos.

En resumen, la participación ciudadana en Colombia demanda transformaciones relevantes en cuanto a visibilidad e información. El acceso, más allá de lo físico, depende de los datos sobre los cuales pueda disponer la ciudadanía durante la toma de decisiones. Estar presente en el debate sin conocer lo que allí se discute es, de cierto modo, estar ausente al mismo tiempo.

\section{¿Cómo se ve el gobierno digital en Colombia?}

Durante la última década, los gobiernos colombianos se han alineado con la tendencia mundial de digitalización. Las administraciones de

- Andrés Pastrana, Álvaro Uribe y Juan Manuel Santos han desarrollado un proceso de transformación del sector público a través del empleo de las TIC con directrices y enfoques que, dependiendo del periodo de gobierno, varían ligeramente. En primera instancia, con el CONPES 3072/2000 y la Directiva Presidencial No. 02 se establecieron las bases sobre las cuales se ha construido la política de gobierno digital en Colombia. Posteriormente, en 2010 se definieron con mayor detalle los puntos clave del e-gobierno (Sanabria et al, 2014).

El programa que encarna la iniciativa de gobierno digital en Colombia es Gobierno en Línea. Su propósito es promover la participación ciudadana, transparencia, cooperación entre entidades estatales y competitividad del país. Hasta el momento, este enfoque ha logrado una suerte de spill over, es decir, una extensión de estas características a otras áreas de manera progresiva. Ello a conseguido que numerosas entidades estatales en todos los niveles de gobierno empleen plataformas digitales para el desempeño de sus funciones (Sanabria et al, 2014)

Entre las iniciativas más destacables, se encuentran datos. gov.co y Urna de Cristal. Aunque gran parte del gobierno digital colombiano cuenta con importantes problemas, estos dos programas destacan por su carácter innovador y por dirigir el e-gobierno del país hacia un avance importante. Datos.gov.co constituye un portal de datos de gobierno abierto, mientras que Urna de Cristal tiene un enfoque de participación ciudadana, lo cual acerca a Colombia a la democracia digital.

Ahora bien, aunque se están dando avances en materia de gobierno digital, la mayoría de iniciativas corresponden a plataformas de carácter informativo en la forma de datos de gobierno abierto. Esto significa que el gobierno digital del país aún se encuentra en la inmadurez y que se halla en la fase de gobernanza digital. La ausencia de espacios de participación digital viables evita que avance hacia el 
punto final de su proceso. De hecho, existen numerosos proyectos que materializan y perpetúan esta realidad, entre los cuales se halla Cali Distrito Especial.

\section{Cali Distrito Especial}

Cali Distrito Especial es el proyecto por el cual el municipio de Santiago de Cali pasa a ser acobijado bajo el estatus de distrito especial. Esta transformación implica la institución de una nueva estructura administrativa, así como una división territorial diferente. Consiste, de manera general, en el reemplazo de las actuales comunas por localidades de mayor tamaño gobernadas por alcaldes locales, con el propósito de facilitar y promover la participación ciudadana en la toma de decisiones concernientes a estas (Alcaldía de Cali, 2019).

El proceso de construcción del proyecto ha sido particular. En términos legales, el proyecto ya está en marcha y la ciudad de Cali debe hacerse distrito prontamente. Para tal efecto, se requerían propuestas de estructuración administrativa y territorial a ser presentadas en el Concejo de la ciudad. Aunque la tarea se desarrolló, para noviembre de 2020 todavía no se han discutido las proposiciones a pesar de que están listas hace meses. Aunque la construcción académica de la propuesta de división territorial contó con espacios de participación ciudadana, estos se limitaron a socialización de ideas ya discutidas y sentadas por los tomadores de decisiones. De igual forma, a pesar de que se recibió la retroalimentación de las personas asistentes a los talleres de la alcaldía, los aportes y datos sustraídos de las intervenciones no tuvieron mayor efecto sobre el resultado final. En síntesis, el problema más relevante de Cali Distrito Especial es que la mayoría de la población caleña desconoce completamente el proyecto. A pesar de los espacios de participación ciudadana abiertos para su socialización, esta realidad sigue siendo prevalente, lo cual reitera la importancia de investigar formas de intervenir sobre este punto.

\section{Discusión}

Con estas ideas puestas sobre la mesa, resulta más sencillo establecer una estructura teórica que soporte la presente investigación. En ese orden de ideas, es posible afirmar que la herramienta a desarrollar se encuentra en el contexto del gobierno digital y busca, en cierta medida, aportar en el avance hacia la fase de democracia digital con Cali Distrito Especial como caso de estudio. Para ello, va a contar con los elementos teóricos asociados con la participación digital y los datos abiertos, no sin dejar de dar importancia a los retos de cada categoría analítica.

Es claro, entonces, que para lograr un mejor acceso más allá de lo físico, resulta fundamental proveer información relevante a los ciudadanos, particularmente, a través del uso de interfaces amigables que permitan apropiar la información con facilidad. Con suficiente conocimiento la ciudadanía no sólo adquirirá herramientas para participar a cabalidad de los procesos deliberativos, sino que podría también difundir la información entre sus pares (Hague y Loader, 2005).

\section{Metodología}

El proyecto se realizó bajo la metodología de diseño del doble diamante. Esto implica un proceso iterativo con fases consecutivas cuyo propósito es alimentar las siguientes etapas del proyecto (Design Council, 2019) Así, en esta iteración del proyecto, se inició con la fase de descubrimiento, con base en los resultados se dio paso a la fase de definición para posteriormente construir y validar la herramienta. El desarrollo de estas fases se distribuyó en dos etapas. La primera, hasta diciembre de 2020, buscó determinar el mayor número de factores contextuales y funcionales posible para la generación de la herramienta; mientras tanto, en la última etapa, se desarrollará un prototipo de esta y será puesto a prueba con ciudadanos y expertos.

De este modo, en la primera y segunda fase de ideación se hizo una revisión de la literatura existente en torno al tema, así como entrevistas semiestructuradas a expertos con el propósito de iluminar los factores relevantes en los mecanismos de participación ciudadana en relación con las nuevas tecnologías. Asimismo, se hizo un recorrido a través de cuatro plataformas de participación ciudadana digital con el propósito de establecer elementos o atributos que puedan ser aplicados a la herramienta que se busca producir a partir de la presente investigación.

Con los resultados de las fases de descubrimiento y definición se dará inicio al desarrollo de la herramienta. En este punto se empleará el co-desarrollo como método de diseño participativo en el que los usuarios participarán en la construcción del mecanismo de participación (Muller, Wildman y White 1993). Así, se generará un 
prototipo de herramienta que será puesto a prueba con ciudadanos y expertos durante varias iteraciones con el fin de mejorarlo y adaptarlo al contexto necesario paulatinamente. Finalmente, previo a la validación del prototipo, se implementará el Scrum como metodología para su desarrollo, lo que complementa a las demás fases. En ese orden de ideas, el proyecto finalizará con la última iteración de la herramienta.

\section{Trabajo de Campo}

Esta es la primera de dos fases del trabajo de campo para la presente investigación. Su propósito, tal y como se relata en la sección de metodología, es el de encontrar elementos y particularidades que permitan determinar la funcionalidad de la herramienta a desarrollar. En este sentido, se hicieron dos entrevistas semiestructuradas y un análisis a profundidad de cuatro herramientas de participación ciudadana digital que podrían arrojar luces sobre las tendencias y características de la e-participación en Colombia.

\section{Lo que dicen los expertos}

Para esta fase del trabajo de campo se entrevistó a Pablo Sanabria Pulido y a Enrique Rodríguez Caporali, ambos académicos involucrados en la formulación de las posibles divisiones territoriales del futuro Distrito de Cali. La indagación se enfocó en aspectos que pudieran dar luces sobre el contexto en el cual se encuentran inmersos los espacios de participación ciudadana, así como sus dinámicas, desafíos y posibilidades. Se le dio particular relevancia a las implicaciones de Cali Distrito Especial sobre la ciudadanía para establecer qué información va a transmitir la herramienta y la modalidad en que lo haría. Asimismo, se hizo énfasis en el proceso de co-creación que hizo la alcaldía junto con la Universidad del Valle, con el propósito de encontrar necesidades que pudieran ser abordadas.

El propósito de Cali Distrito Especial, según los entrevistados, es desconcentrar las funciones que tiene la alcaldía y facilitar la interacción entre ciudadanos y tomadores de decisiones. En ese sentido, el proyecto busca, además de dividir la ciudad de una forma distinta, crear estructuras administrativas más localizadas con el objetivo de generar una comunicación más cercana y responsiva. Entre los elementos más importantes de Cali Distrito Especial se encuentran los planes de desarrollo locales, los cuales serán definidos en conjunto con las comunidades a las cuales afecten. En este orden de ideas, la herramienta digital que plantea la presente investigación tiene la capacidad de ser un primer acercamiento a este tipo de discusión local o, quizás, puede sentar las bases para futuras investigaciones relacionadas con el tema.

El proceso de construcción colectiva realizado por la alcaldía no fue muy exitoso. Aunque se hicieron talleres con líderes y

ciudadanos, estos fueron tan sólo informativos y los aportes hechos durante los mismos no tuvieron mayor impacto sobre el proyecto más allá de pequeñas interacciones que sirvieron a los expertos para contextualizarse mejor. Aunque el mandato de los talleres y espacios de discusión no era consultivo, sino de socialización, la conversación con los expertos sugiere que de cualquier modo salieron mal y no cumplieron a cabalidad con su propósito.

En cuanto a las dinámicas particulares de la participación ciudadana, lo dicho por los entrevistados soporta la literatura existente sobre el tema. En efecto, tanto Enrique Rodríguez Caporali como Pablo Sanabria mencionaron que los espacios de participación ciudadana en Colombia suelen ser convertidos en espacios de desahogo sin mayor efecto sobre la realidad de los ciudadanos. Ello, como producto de la deplorable comunicación del Estado, así como el reducido tiempo de las personas para participar. En ese orden de ideas, los espacios de participación ciudadana se encuentran limitados a las determinaciones de los gobiernos que los abren. Así, son estos quienes establecen las condiciones de lo que se discute, la escala y las invitaciones a los líderes que ellos consideran pertinentes para la discusión. Estos últimos también son un elemento relevante. Como la participación ciudadana es un elemento de difícil acceso, es común que surjan líderes comunitarios autoproclamados, sin necesariamente tener respaldo popular o ser representativos del grupo al que dicen estar adscritos.

En consecuencia, existen pocos grupos y sectores organizados para la discusión de políticas públicas. En el caso de Cali Distrito Especial tan sólo las zonas rurales y los ambientalistas lograron organizarse para consolidar un frente unido. Ahora bien, el problema de la información se mantiene vigente para estos casos: cuando los académicos intentaban contextualizarlos, carecían de la suficiente credibilidad para influenciar las decisiones. 
Acerca del estado de las plataformas de participación ciudadana digital Las aplicaciones analizadas corresponden en su mayoría a iniciativas a nivel ciudad en el contexto colombiano. Esto como respuesta a la necesidad de producir una herramienta con características consistentes con las prácticas y dinámicas de la participación ciudadana en el país. Tal decisión no implica un descarte total de iniciativas de otras características, pero las deja por fuera durante este primer momento.

El análisis de las plataformas se desarrolla a la luz de cuatro categorías. La navegabilidad, entendida como la facilidad con la cual los ciudadanos pueden acceder a los proyectos o temáticas de discusión dispuestas en las plataformas; el funcionamiento de la participación, entendido como la dinámica bajo la cual los ciudadanos realizan aportes o expresan su opinión respecto a las temáticas de discusión; el nivel de la participación, entendido como el contexto en el que se ubican los proyectos, ya sea país, ciudad, comuna o barrio; finalmente, la continuidad, que busca evaluar si las interacciones con los proyectos son únicas o si se mantienen durante un período de tiempo hasta que culminen.

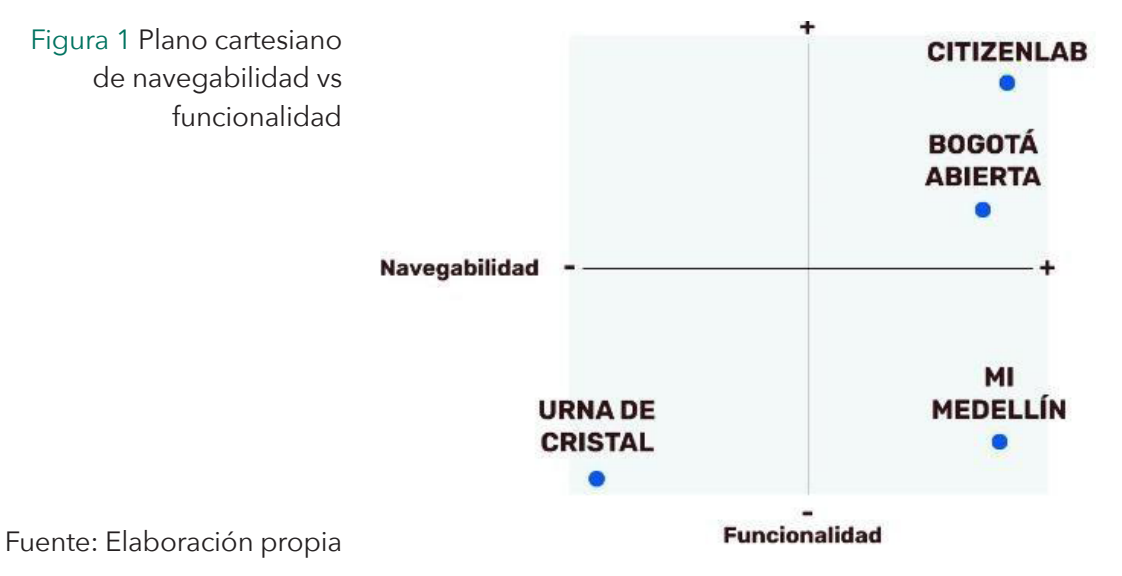

\section{Urna de Cristal}

Cuando se revisa la literatura sobre la participación ciudadana digital en Colombia, Urna de Cristal suele destacar entre las iniciativas existentes. En un país donde este tipo de prácticas aún es incipiente, esta se perfila como una herramienta de gran relevancia por ser pionera en la búsqueda de la democratización en la participación. No obstante, en términos más específicos, cuenta con numerosos vacíos que limitan su alcance. Cuando los usuarios se acercan a la plataforma encuentran que su navegabilidad es reducida. La arquitectura de la información y el proceso de navegación hace que los procesos participativos carezcan de protagonismo. Aunque el propósito de la página es primordialmente la participación digital ciudadana, los espacios se encuentran en una subsección que resalta muy poco.

El funcionamiento de la participación presenta problemas similares al de los espacios tradicionales. En sí, el involucramiento ciudadano es reducido y cuando se da suele ser para expresar dudas con respecto a cargas administrativas y procedurales. Los ciudadanos interactúan para pedir explicaciones sobre el funcionamiento de trámites para acceder a ingreso solidario, jóvenes en acción y otras ofertas gubernamentales que las entidades estatales deberían ser capaces de solucionar en las dependencias correspondientes. Lo regular en esta aplicación es que se traten temas de carácter general y no proyectos como tal. Es por esto que el nivel de la participación podría ubicarse en lo nacional o mantenerse como inconcluso.

La dinámica bajo la cual funciona la aplicación consiste en que se hayan preguntas dispuestas por los tomadores de decisiones durante un período de tiempo determinado. Los ciudadanos pueden responder a estas y hacer aportes como deseen. En sí mismo, este funcionamiento no da pie para que haya continuidad, pues los usuarios pueden dejar su comentario y nunca más volver a involucrarse. De manera análoga, una vez cerrado el período de respuesta, el tomador de decisiones no tiene cómo reportar avances o transformaciones en la agenda.

\section{Mi Medellín}

Mi Medellín es una plataforma desarrollada por Ruta N, el centro de negocios e innovación de Medellín. Tiene como propósito abrir la participación ciudadana en la ciudad a través de las TIC. Su navegabilidad es directa, con tan sólo un click se puede acceder a los procesos de participación ciudadana dispuestos para la discusión. El funcionamiento de la participación es diferente a la que se da en Urna de Cristal. En la plataforma están dispuestos varios temas y proyectos que a su vez cuentan con actividades o pasos que son votados uno a uno para cada fase del proyecto. La participación se da a nivel de ciudad y cuenta con información robusta sobre la iniciativa, así como palabras clave que permiten trazar con mayor facilidad el proceso. Del mismo modo, los proyectos están divididos en fases que se informan claramente a la ciudadanía, lo cual facilita una continuidad en el desarrollo de los procesos. 


\section{Bogotá Abierta}

Bogotá Abierta es similar a Mi Medellín en cuanto a que está construid para albergar proyectos de nivel ciudad bajo una dinámica similar. La navegación de la plataforma es directa, cuenta con gran claridad con respecto al funcionamiento de la participación y cuenta con varias funcionalidades que cambian dependiendo del propósito que busque el tomador de decisiones. En algunos casos los proyectos están abiertos a aportes escritos por los ciudadanos y en otros, son simplemente abiertos para votación de actividades en orden de preferencia.

\section{Citizenlab}

De todas las aplicaciones mencionadas con anterioridad, Citizenlab es la más robusta. Esta última fue producida en Europa y se ha perfilado como una de las plataformas de participación ciudadana digital más famosas del mundo. A pesar de que no se encuentra en el contexto colombiano, su papel de referente internacional para este tipo de iniciativas hace valioso analizarla. Citizenlab cuenta con una navegación sencilla. Los proyectos se encuentran dispuestos en la primera página y es claro cómo participar en ellos Entre los elementos más destacables de su funcionamiento se hallan las numerosas funcionalidades con las que cuenta. Así como Bogotá Abierta, esta también tiene la posibilidad de abrir los proyectos para votación, lluvia de ideas e información. El nivel de la participación es flexible, pues la dinámica facilita el filtrado de proyectos por ubicación geográfica y, asimismo, facilita una continuidad en los proyectos y su discusión.

\section{Análisis: Diferencias y semejanzas entre las aplicaciones}

Entre las herramientas analizadas, el nivel de participación es, en cierta medida, común a todas. Aunque es valioso hacerlo, la literatura y las palabras de los expertos consultados llevan a una consideración distinta, ya que la apuesta apunta a aproximaciones más localizadas y de carácter comunitario que podrían tener más éxito o arrojar distinciones sobre las cuales construir. Entre las cuatro herramientas tan sólo Citizenlab tiene la facultad de cambiar la escala de la participación ciudadana y aun así no está diseñada alrededor de esta posibilidad. Si bien la presente investigación se enfoca en la transmisión de información, dar pequeños pasos con respecto a la dinámica de la participación podría facilitar o mejorar futuras consultas sobre el mismo tema. Así, el trabajo de campo desarrollado permite entrever a necesidad de producir una herramienta digital contextualizada y

que corresponda a los retos del contexto colombiano, no sin dejar de lado las funcionalidades que pueden darle versatilidad, a pesar de que pertenezcan a aplicaciones distintas.

\section{- Oferta de valor: Fractal}

A partir de los elementos teóricos y empíricos recolectados durante la investigación, se llegó a la conclusión de que resulta fundamental, al menos para el caso colombiano, generar herramientas de participación ciudadana digital con un enfoque diferencial comunitario. En otras palabras, es imperativo alejarse del paradigma con el que operan las herramientas de participación ciudadana en otros contextos, pues se basan en procesos de mayor escala y diferentes características. En contraste con dichas experiencias, en el contexto colombiano demanda la necesidad de construir procesos alrededor de las comunidades con el objetivo de facilitar el acceso a los espacios de participación, así como a la información pertinente.

De tal manera, Fractal surge como una referencia a los fractales, estructuras cuyas partes terminan por repetirse en diferentes escalas. Dicha metáfora hace referencia al funcionamiento de la participación ciudadana en sus diferentes escalas. Las discusiones a nivel de comuna y de barrio pueden tener tanto o incluso más efecto que aquellas generadas a nivel ciudadano. Así, la plataforma cuenta con una serie de elementos que materializan las condiciones necesarias para cumplir con el objetivo del proyecto.

\section{Determinantes de diseño}

Los determinantes de diseño se componen de cinco términos: Enfoque local, iniciativa ciudadana, enfoque informativo, liderazgos ciudadanos, continuidad de procesos. El enfoque local es la herramienta que debe garantizar que la información transmitida a los ciudadanos tenga un enfoque territorial. Para Cali Distrito Especial, es ideal que la nueva división administrativa sea determinada en el contexto de los barrios o las comunas. En cuanto a la iniciativa ciudadana, se puede definir como la herramienta que, una vez definido el enfoque local, debe permitir al ciudadano iniciar contactos con tomadores de decisiones y otros 
ciudadanos. Asimismo, debe darle un alto grado de libertad sobre la gestión de su participación. Con relación al enfoque informativo, uno de los mayores propósitos de la herramienta es informar para participar. En ese sentido, es fundamental que tanto tomadores de decisiones como ciudadanos reciban información clara y concisa a través de la herramienta. Los liderazgos ciudadanos se pueden definir como el derecho de las comunidades a elegir los liderazgos y representaciones que se involucren con mayor profundidad en el proceso. Por último, la continuidad de procesos hace referencia a que, de ser necesario, los ciudadanos y tomadores de decisiones tendrán la oportunidad de dividir los proyectos y actividades en etapas con períodos de tiempo delimitados.

\section{Requerimientos de diseño}

Los requerimientos de diseño se dividen en funcionales y tecnológicos. Los funcionales se resumen en nueve postulados. El primero de ellos: (1) Plantea que el sistema contará con un clasificador que presente al ciudadano los proyectos disponibles para discutir en su comuna; (2) asimismo, el sistema permitirá al ciudadano interactuar con otros ciudadanos a través de salas de discusión virtuales; (3) de ser requerido, el sistema contará con la oportunidad de abrir proyectos y dividirlos en diferentes etapas; (4) el sistema contará con recordatorios que anuncien a los ciudadanos momentos importantes del proceso deliberativo (nueva información, fechas de votación, entre otras); (5) el sistema permitirá a los tomadores de decisiones y ciudadanos etiquetar los diferentes proyectos bajo categorías que puedan ser utilizadas para su búsqueda; (6) respecto a sus funcionalidades, el sistema contará con la posibilidad de abrir proyectos o temáticas con formatos como lluvia de ideas, votación o discusión general; (7) de igual manera, con relación al tema de las funcionalidades, el sistema contará con la posibilidad de que los ciudadanos inicien proyectos y distribuyan tareas entre ellos; (8) el sistema permitirá que los ciudadanos a nivel barrial o comunal se postulen y elijan líderes de la comunidad para que guíen el proceso o incluso elijan no contar con un líder determinado; (9) por último, la herramienta deberá tener una funcionalidad para agregar la información recolectada y visualizarla de manera clara. En relación con los requerimientos tecnológicos, estos se resumen en dos postulados. En primer lugar, la herramienta deberá ser desarrollada para formato móvil a modo de garantizar el acceso fácil y rápido a sus funciones. En adición a lo anterior, esta no podrá consumir mucha memoria con el propósito de - evitar la posibilidad de que ciertas personas se queden por fuera de esta por no tener un hardware que soporte software de gran envergadura. Por otro lado, se encuentran las restricciones de diseño que hacen referencia específicamente a los marcos legales bajo los que se debe regir la plataforma. A grandes rasgos, se puede decir que: El sistema se desarrollará en concordancia con lo estipulado en la Ley 1933 de 2018, la Ley 1617 de 2013 y la Ley 1581 de 2012.

\section{Funcionamiento}

\section{Ciudadano}

El funcionamiento de la plataforma depende del tipo de usuario. En primer lugar, para el caso del ciudadano, al ingresar a la aplicación después de registrarse o iniciar sesión, este se encontrará con un listado de los proyectos disponibles en su barrio. Posteriormente, tendrá la oportunidad de afiliarse a uno. Al hacerlo, recibirá información en formato de video y audio antes de estar en capacidad de entrar a votar, discutir o proponer. Del mismo modo, tendrá la oportunidad de complementar la información ya disponible, así como de comunicarse con los tomadores de decisiones disponibles a través de mensajes. En contraste, el tomador de decisiones tendrá la posibilidad de visualizar la información recopilada de forma agregada. En su inicio aparecerán las solicitudes de los ciudadanos, así como los procesos en los que está involucrado y mensajes asociados con estos. Del mismo modo, el tomador de decisiones podrá avanzar las etapas de un proyecto si resulta necesario. Con relación a dicha actividad, se transmitirán notificaciones a los ciudadanos involucrados. Este tipo de perfil, también tendrá la oportunidad de actualizar la información y deliberar con los ciudadanos.

$$
\begin{array}{r}
\text { Figura } 2 \text { Funcionamiento } \\
\text { de Fractal }
\end{array}
$$

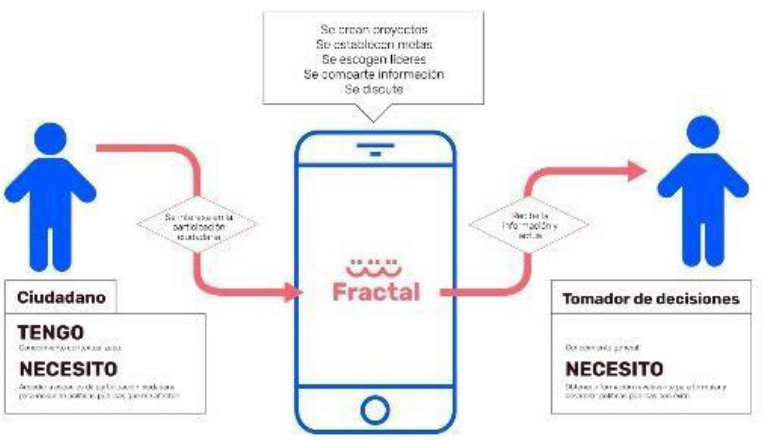




\section{Conclusiones}

La presente investigación cuenta con resultados de carácter técnico y teórico que podrían contribuir a la discusión sobre la generación e implementación de herramientas digitales en las diferentes etapas del ejercicio democrático. La introducción de plataformas digitales tiene la facultad de agenciar al ciudadano para que gestione su participación en los diferentes espacios democráticos, no sólo en términos de acceso a las discusiones, sino a la información que las rodea. Así mismo, abre la puerta a un mapeo más claro de los actores y partes interesadas, sus motivaciones, su comportamiento y su modelo de interacción; con ello abriendo la puerta a consensos y relaciones tradicionalmente limitadas por las características de los mecanismos existentes.

El funcionamiento y desempeño de herramientas digitales como Fractal tiene la capacidad de elevar ideas sobre el gobierno digital y sus implicaciones en Colombia e incluso América Latina, un tema que aún se encuentra en etapas tempranas de desarrollo y no ha sido abordado con la suficiente profundidad por la academia y el Estado. La aplicación de plataformas digitales que escapen el enfoque técnico, que hoy es dominante, contribuye al avance de soluciones tecnológicas basadas en el contexto y particularidades de la realidad colombiana.

Es necesario resaltar que trasladar los elementos de la participación presencial a un espacio digital no es una condición suficiente para superar los problemas que rodean a la participación ciudadana en Colombia. Resulta fundamental pensar en la implementación de cualquier plataforma como un complemento a las experiencias presenciales, pues ambas suplen la inclusión de segmentos distintos de la población y cuentan con limitaciones diferentes que sólo pueden ser abordadas si se acompañan ambas modalidades.

De este modo, se puede afirmar que las características de las herramientas de participación ciudadana digital deben variar dependiendo de la escala en la cual se dan las discusiones. Así, proyectos de nivel comunal, barrial, ciudadano o distrital operarán de forma diferencial entre ellos. Por otro lado, en el momento de elegir para quién se diseña la herramienta, es de gran importancia tener en consideración que existen dos posibilidades; en la participación ciudadana tradiciona es usual que sean las personas mayores quienes se involucren como resultado del tiempo que tienen en sus manos. Sin embargo, con la variable tiempo fuera de la ecuación gracias al carácter digital de la plataforma a desarrollar, es posible que se involucren los jóvenes, teniendo en cuenta que son la población con mayor interés en participar (Velásquez et al, 2019b). En ese sentido, es importante diseñar para la población con mayores necesidades.

Finalmente, es importante también dar relevancia a

- la imposibilidad de solucionar la totalidad de problemas y conflictos que plagan a la participación ciudadana en el contexto colombiano con la introducción de plataformas digitales. Más allá de los mencionados beneficios, existen factores políticos como la corrupción, el clientelismo, la falta de comunicación interinstitucional, la existencia o carencia de voluntad política y la incidencia de corrientes ideológicas; elementos que pueden generar fricciones y resistencia a las plataformas incluso antes de su introducción. L mera existencia de una plataforma sólo puede garantizar la apertura de nuevos espacios democráticos, la visibilización de actores y de problemáticas en diversas escalas.

Referencias bibliográficas

Alcaldía de Cali. (2019). Documento Informe Final de Participación Ciudadana. Recuperado de: https://www.cali.gov.co/ participacion/publicaciones/148724/plan-municipal-departicipacion-ciudadana/

Bolgherini, S. (2007). The Technology Trap and the Role of Political and Cultural Variables: A Critical Analysis of the E-Government Policies. Review of Policy Research, 24(3), 259-275. doi:10.1111/ j.1541-1338.2007.00280.x

DANE. (2019). Encuesta de Cultura Política. Recuperado de: https:// www.dane.gov.co/index.php/estadisticas-por-tema/cultura/ cultura-politica-encuesta

Design Council. (2019). What is the framework for innovation? Design Council's evolved Double Diamond. Design Council. Recuperado de: https://www.designcouncil.org.uk/news-opinion/whatframework-innovation-design-councils-evolved-double-diamond

Dunleavy, P., Margetts, H., Bastow, S., \& Tinkler, J. (2006). New public management is deadlong live digital-era governance. Journal of public administration research and theory, 16(3), 467-494. 
Hague, B. N., \& Loader, B. D. (Eds.). (2005). Digital democracy: Discourse and decision making in the information age. Londres: Routledge. Holzer, M., Melitskin, J., Rho, S. Y., \& Schwester, R. (2004). Restoring trust in government: The potential of digital citizen participation. Frontiers of Public Administration, 6, 6-23.

Ingrams, A., Piotrowski, S., \& Berliner, D. (2020). Learning from Our Mistakes: Public Management Reform and the Hope of Open Government. Perspectives on Public Management and Governance, 3(4), 257-272.

Kardan, A. A., \& Sadeghiani, A. (2011). Is e-government a way to e-democracy? Government Information Quarterly, 28(4), 466-473. DOI:10.1016/j.giq.2010.12.007

Muller, M. J., Wildman, D. M., \& White, E. A. (1993). Taxonomy of PD practices: A brief practitioner's guide. Commun. ACM, 36(6), 26-28.

Netchaeva, I. (2002). E-government and e-democracy: a comparison of opportunities in the north and south. Gazette (Leiden, Netherlands), 64(5), 467-477.

Open Data Charter (2015). Principles: International Open Data Charter. Recuperado de: https://opendatacharter.net/principles/

Peña-López, I. (2014). UN e-Government Survey 2014. E-Government for the Future We Want.

Sanabria, P., Pliscoff, C., \& Gomes, R. (2014). E-government practices in South American countries: Echoing a global trend or really improving governance? The experiences of Colombia, Chile, and Brazil. In Open Government (pp. 17-36). Springer, New York, NY.

Shackleton, P., Fisher, J., \& Dawson, L. (2004). Evolution of local government e-services: the applicability of e-business maturity models. 37th Annual Hawaii International Conference on System Sciences, 2004. Proceedings of The. DOI:10.1109/ hicss.2004.1265308

Thomas, J. \& Streib, G. (2003). The New Face of Government: CitizenInitiated Contacts in the Era of E-Government. Journal of Public Administration Research and Theory: J-PART, 13(1), 83-101.

Velásquez, F., \& González, E. (2003). ¿Qué ha pasado con la participación ciudadana en Colombia? Bogotá: Fundación Corona.

Velásquez, F., González, E., Martínez, F. P., Peña N.J., Arévalo P. J., Vargas, R. J. (2019a). ¿Qué ha pasado con la participación ciudadana en Colombia? 2003 - 2018.
Tomo 1. Bogotá: Foro Nacional por Colombia.

(2019b). ¿Qué ha pasado con la participación ciudadana en Colombia? 2003 - 2018

Tomo 2. Bogotá: Foro Nacional por Colombia.

(2019c). ¿Qué ha pasado con la participación ciudadana en Colombia? $2003-2018$.

Tomo 3. Bogotá: Foro Nacional por Colombia.

Yu, H., \& Robinson, D. G. (2011). The new ambiguity of open government. UCLA L. Review Discourse, 59, 178-190. 

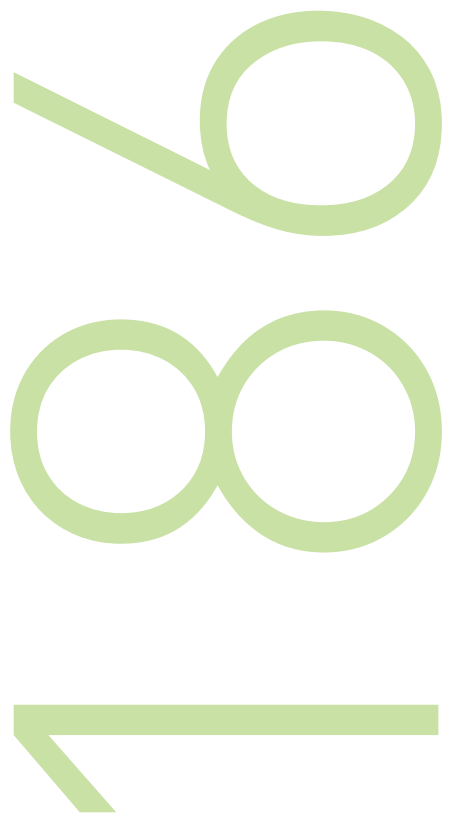